\title{
Surgical Techniques at Cesarean Delivery: A U.S. Survey
}

\author{
Deirdre J. Lyell ${ }^{1}$ Michael Power $^{2}$ Katie Murtough ${ }^{2}$ Amen Ness ${ }^{1}$ Britta Anderson ${ }^{2}$ Kristine Erickson ${ }^{2}$ \\ Jay Schulkin ${ }^{2}$
}

${ }^{1}$ Department of Obstetrics and Gynecology, Stanford University, Palo Alto, California

2 Research Department, American College of Obstetricians \& Gynecologists, Washington, District of Columbia

\begin{abstract}
Address for correspondence Deirdre J. Lyell, MD, Department of Obstetrics and Gynecology, Stanford University, 300 Pasteur Drive, MC 5317, Palo Alto, CA 94305 (e-mail: dlyell@stanford.edu).
\end{abstract}

Surg J 2016;2:e119-e125.

\begin{abstract}
Keywords

- cesarean

- survey

- surgical technique

- surgical closure

Objective To assess the frequency of surgical techniques at cesarean delivery (CD) among U.S. obstetricians.

Methods Members of the American College of Obstetrician Gynecologists were randomly selected and e-mailed an online survey that assessed surgical closure techniques, demographics, and reasons. Data were analyzed using SPSS (IBM Corp., Armonk, New York, United States), descriptive statistics, and analysis of variance.

Results Our response rate was $53 \%$, and 247 surveys were analyzed. A similar number of respondents either "always or usually" versus "rarely or never" reapproximate the rectus muscles ( $38.4 \%$ versus $43.3 \%, p=0.39$ ), and close parietal peritoneum $(42.5 \%$ versus $46.9 \%, p=0.46)$. The most frequently used techniques were double-layer hysterotomy closure among women planning future children (73.3\%) and suturing versus stapling skin (67.6\%); the least frequent technique was closure of visceral peritoneum (12.2\%). Surgeons who perform double-layer hysterotomy closure had fewer years in practice (15.0 versus 18.7 years, $p=0.021$ ); surgeons who close visceral peritoneum were older ( 55.5 versus 46.4 years old, $p<0.001$ ) and had more years in practice ( 23.8 versus 13.8 years practice; $p<0.001$ ).

Conclusion Similar numbers of obstetricians either reapproximate or leave open the rectus muscles and parietal peritoneum at $C D$, suggesting that wide variation in practice exists. Surgeon demographics and safety concerns play a role in some techniques.
\end{abstract}

Following a nearly continuous rise since 1996, cesarean delivery (CD) remains the most common major surgery performed among women in the United States, at a rate of $32.8 \%$ in $2012 .^{1}$ Despite the high frequency of $\mathrm{CD}$, few high-quality data exist regarding the effects of different surgical techniques on maternal or neonatal morbidity, or the frequency of their use. Some surgical closure techniques at $C D$, such as closure of the parietal and visceral peritoneum, have been shown to influence short-term outcomes such as increased operative time, ${ }^{2}$ increased infectious morbidity with closure of the visceral peritoneum, ${ }^{3}$ and long-term outcomes such as adhesions and their consequences. ${ }^{4-8}$ Techniques such as rectus muscle reapproximation are not typically mentioned in studies, ${ }^{9-11}$ and it is unknown how frequently they are used at CD.

Anecdotal evidence suggests that a large variation in surgical closure techniques exists among obstetricians, particularly for techniques such as rectus muscle reapproximation. A European survey identified that a large practice variation exists for most surgical techniques used at $C D$, with the exception of double-layer hysterotomy closure, a technique that was practiced by most. ${ }^{12} \mathrm{~A}$ recently published Canadian survey of hysterotomy closure techniques at $\mathrm{CD}$ also received

March 23, 2016 accepted after revision October 13, 2016
Dol http://dx.doi.org/

10.1055/s-0036-1594247. ISSN 2378-5128.
Copyright (c) 2016 by Thieme Medical Publishers, Inc., 333 Seventh Avenue, New York, NY 10001, USA. Tel: +1(212) 584-4662.
License terms

(1) (1) $\Theta \circledast$ 
revealed a high frequency of double-layer hysterotomy closure. ${ }^{13}$ Although a growing number of randomized controlled trials have been published regarding surgical techniques at $\mathrm{CD}$ and short-term outcomes, ${ }^{9,11}$ little is known about what obstetricians actually do in practice in the United States. It is important to identify which surgical techniques are used at CD in current practice, in concert with studies that identify which techniques should be used in best practice.

We surveyed U.S. obstetricians to assess the frequency of surgical closure techniques used at $\mathrm{CD}$, and identified the variation in practice and the reasons for choosing each technique.

\section{Methods}

One thousand members of American College of Obstetrician Gynecologists (ACOG) were randomly selected and invited to participate in this study between April and June of 2014. Two hundred of the participants were members of the Collaborative Ambulatory Research Network (CARN), a network of ACOG members who have agreed to participate in research conducted by ACOG. Participants were contacted by e-mail with basic information about the study and an electronic link to access the survey. E-mail recipients who do not practice obstetrics were given a link to opt out. Obstetricians who perform $C D$ and completed at least $60 \%$ of the survey were included. A total of six reminder e-mails were sent to those who had not opened or responded to the survey, each approximately within 1 week of each other. A follow-up letter was sent via mail to those who had not opened any of the emails and to those who had opened an e-mail but had not yet responded ( $n=699$ ) at the time of the fifth e-mail reminder.

The survey consisted of 19 routine questions about respondent demographics and 25 questions about surgical techniques. The surgical techniques questions are summarized in -Fig. 1.

CARN Fellows (1,248 members as of February 2015) are a group of ACOG Fellows and Junior Fellows in Practice (JFPs) who volunteer to participate in survey studies on an ongoing basis. CARN Fellows are a nationally representative sample of the age, sex, and geographic distribution of ACOG Fellows and JFPs, with proportionate representation from all 11 ACOG districts. Assessments of knowledge and practice between CARN Fellows and ACOG Fellows not in the CARN have shown they do not differ. An examination of 17 randomly selected studies showed few differences in responses between CARN and non-CARN ACOG study participants, with a mean correlation of $r=0.99(p<0.001)$ for the responses to ordinal questions (e.g., 1- to 5-point scales). Although ACOG cannot exclude the possibility that CARN Fellows differ in aspects of knowledge, training, or practice in some areas of clinical medicine, to date that has not been shown to be true. ACOG continues to test for differences every year.

Data were analyzed using IBM SPSS Statistics 20.0 (IBM Corp., Armonk, New York, United States). For the purposes of data analysis, the responses "always" and "usually" were combined and considered to be commonly performed surgical techniques, and "rarely" and "never" were combined and considered to be techniques not commonly performed. Chi-squared and Fisher exact tests were used where relevant, and analysis of variance analyses were used to measure group differences; $p<0.05$ was considered statistically significant.

This study was reviewed by the Institutional Review Board and Stanford University and was determined to be exempt.

\section{Results}

Of the 1,000 obstetrician-gynecologists invited to participate, 48 did not have valid e-mail addresses. Of the remaining 952, 706 opened at least one e-mail and/or confirmed receipt of the follow-up letter; 146 responded that they do not perform CDs or had retired from practice and were considered ineligible to participate. The final sample included 560 potentially eligible responders, of whom 295 participated in the online survey for a 53\% total response rate (70\% CARN, $48 \%$ non-CARN). Of the 295 responses, 48 were incomplete, defined by less than $60 \%$ of the survey completed. Data analyses reflect the responses of 247 participants (44\% complete response rate).

Demographics for the survey respondents are shown in -Table 1. The cohort was $63.6 \%$ women, $86.6 \%$ generalist obstetrician-gynecologists, with an average age of 48.4 (standard error of the mean, \pm 0.7 ), and average years in practice of 16.1 (standard error of the mean, \pm 0.7 ). The largest group (37\%) were from large ( $\geq 5$ surgeons) practices, $18 \%$ were from university or teaching practices, and $12 \%$ were in solo practice.

The frequencies of surgical techniques used are shown in -Table 2. The most frequently used techniques were double-layer hysterotomy closure among women planning future children (73.3\%), and suturing of the skin (67.6\%); the least frequently used technique was closure of the visceral peritoneum (12.2\%). Wide variation was seen in the techniques of rectus muscle reapproximation and closure of the parietal peritoneum, with similar numbers reporting that they "always or usually" versus "rarely or never" reapproximate the rectus muscles ( $38.4 \%$ versus $43.3 \%, p=0.39$ ), and "always or usually" versus "rarely or never" close the parietal peritoneum ( $42.5 \%$ versus $46.9 \%, p=0.46$ ).

Demographic differences were identified based on practice choices (-Table 3 ). Surgeons who were more likely ("always" or "usually") to perform a single-layer hysterotomy closure for women planning future children had more years in practice compared with surgeons who were less likely ("rarely" or "never") to do so (18.7 versus 15.0 years in practice, $p=0.02$ ). Surgeons who were more likely to close the parietal peritoneum were older, with more years in practice, compared with surgeons who were less likely to do so (50.0 versus 46.5 years old, $p=0.009 ; 17.6$ versus 14.1 years in practice, $p=0.012$ ), and similar patterns were seen among those who close the visceral peritoneum ( 55.5 versus 46.4 years old, $p<0.001 ; 23.8$ versus 13.8 years in practice, $p<0.001$ ) and among those who reapproximate the rectus muscles ( 49.4 versus 46.8 years old, $p=0.052 ; 17.2$ versus 14.5 years in practice, $p=0.055$ ). Surgeons who were more likely to suture the skin had fewer years in practice (15.1 versus 19.2 years, $p=0.022$ ), and no differences were found among those who were more likely to staple the skin. 
Surgical closure technique questions:

1. Do you perform single layer (hysterotomy) closure in women anticipated to have more children?

2. Do you reapproximate rectus muscles in women with singleton pregnancies?

3. Do you close the visceral peritoneum ('bladder flap')?

4. Do you close the parietal peritoneum?

5. Do you suture skin at primary cesarean?

5. Do you staple skin at primary cesarean

Answer choices: always/usually/sometimes/never

Patient request questions:

Do you reapproximate rectus muscles if the patient requests?

Answer choices: yes/no/sometimes

Do you suture skin at primary cesarean if the patient requests?

Answer choices: yes/no

Do you staple skin at primary cesarean if the patient requests?

Answer choices: yes/no

Top three reasons for closure technique questions:

Please indicate the top three reasons you always/usually/sometimes/never:

1. perform a single layer closure in women anticipated to have future children

2. reapproximate rectus muscles

3. close the visceral peritoneum

4. close the parietal peritoneum

5. suture the skin at primary cesarean

6. staple the skin at primary cesarean

Answer choices for each category:

\begin{tabular}{|c|c|c|c|c|}
\hline & \begin{tabular}{c|c}
$\# 1$ \\
reaso
\end{tabular} & $\begin{array}{c}\# 2 \\
\text { reason }\end{array}$ & $\begin{array}{c}\# 3 \\
\text { reason }\end{array}$ & $\begin{array}{c}\text { not a top } 3 \\
\text { reason }\end{array}$ \\
\hline It is how I was taught & 0 & 0 & 0 & 0 \\
\hline Better surgical result & 0 & 0 & 0 & 0 \\
\hline Negative patient experience from doing this & 0 & 0 & 0 & 0 \\
\hline $\begin{array}{l}\text { Depends on whether she will undergo trial } \\
\text { of labor after cesarean (TOLAC) }\end{array}$ & 0 & O & ○ & 0 \\
\hline Concerns for future uterine rupture & 0 & 0 & 0 & 0 \\
\hline Concerns for hemostasis & 0 & 0 & 0 & 0 \\
\hline Concerns for increasing future adhesions & 0 & 0 & 0 & 0 \\
\hline Increases immediate postoperative morbidity & 0 & 0 & 0 & 0 \\
\hline Decreases immediate postoperative morbidity & 0 & 0 & 0 & 0 \\
\hline Based on what appears best at surgery & 0 & 0 & 0 & 0 \\
\hline Saves time & 0 & 0 & 0 & 0 \\
\hline It is evidence-based & 0 & 0 & 0 & 0 \\
\hline
\end{tabular}

Importance questions.

In general, to prevent adhesions, how important is:

1. double-layer hysterotomy closure?

2. reapproximation of rectus muscles?

3. closure of the visceral peritoneum?

4. closure of parietal peritoneum?

Answer choices:

unimportant/slightly unimportant/neutral/slightly important/important

Fig. 1 Summary of survey questions.

We asked surgeons to list their top three reasons for choosing each technique. "Evidence-based" was cited in the top three for a surgeon's choice regarding single-layer hysterotomy closure, visceral peritoneum closure, and suturing of the skin (-Table 4). Surgeons who chose "evidence-based" as a top three reason for their practice were less likely to close the parietal peritoneum (75.9\%) or visceral peritoneum (98.3\%). "It was how I was taught" was frequently a top three reason, cited for a surgeon's decision regarding his or her choice for single-layer hysterotomy closure, and decisions to close the parietal peritoneum, visceral peritoneum, and rectus muscles, without any single technique being performed more frequently. Surgeons who chose "better surgical result" as a top three reason were more likely to close the parietal peritoneum ( $71.6 \%$ versus $17.1 \%$ who did not give this response as a reason, $p<0.001)$ and reapproximate rectus muscles (61.8\% versus $6.1 \%$ who did not give this response as a reason $(p<0.001))$. What "Appears best at time of surgery" 
e122 Surgical Techniques at Cesarean Delivery Lyell et al.

Table 1 Demographics of respondents ${ }^{\mathrm{a}}$

\begin{tabular}{|l|l|l|}
\hline & & $\boldsymbol{n}$ \\
\hline Male (\%) & 36.4 & 90 \\
\hline Female (\%) & 63.6 & 157 \\
\hline Average age (y; mean \pm SEM) & $48.4 \pm 0.7$ & 246 \\
\hline Average years in practice (mean \pm SEM) & $16.1 \pm 0.7$ & 246 \\
\hline Generalist (\%) & 86.6 & 214 \\
\hline Maternal-fetal medicine (\%) & 6.9 & 17 \\
\hline Small (2-4) private partnership (\%) & 27.5 & 68 \\
\hline Large ( $\geq 5$ private partnership (\%) & 37.2 & 92 \\
\hline Solo practice (\%) & 11.7 & 29 \\
\hline Laborist/hospitalist (\%) & 3.6 & 9 \\
\hline University/teaching institution (\%) & 18.2 & 45 \\
\hline More than 50\% private insurance in practice (\%) & $58.3 \pm 1.9$ & 239 \\
\hline More than 50\% public insurance in practice (\%) & $32.7 \pm 1.6$ & 239 \\
\hline More than 50\% uninsured in practice (\%) & $5.2 \pm .5$ & 239 \\
\hline
\end{tabular}

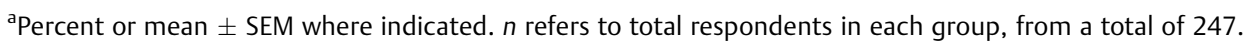

SEM, standard error of the mean.

Table 2 Frequencies of reported surgical techniques, n (\%)

\begin{tabular}{|l|l|l|l|l|}
\hline & “Always" or “Usually" & "Sometimes" & "Rarely" or “Never" & No answer \\
\hline $\begin{array}{l}\text { Single-layer hysterotomy closure if more } \\
\text { children planned }\end{array}$ & $39(15.8)$ & $22(8.9)$ & $181(73.3)$ & $2(2.0)$ \\
\hline Close the parietal peritoneum & $105(42.5)$ & $23(9.3)$ & $116(46.9)$ & $3(1.2)$ \\
\hline Close the visceral peritoneum & $30(12.2)$ & $14(5.7)$ & $194(78.5)$ & $9(3.6)$ \\
\hline Reapproximate the rectus muscles (singletons) & $95(38.4)$ & $41(16.6)$ & $107(43.3)$ & $4(1.6)$ \\
\hline Suture the skin & $167(67.6)$ & $31(12.6)$ & $43(17.4)$ & $6(2.4)$ \\
\hline Staple the skin & $56(22.7)$ & $39(15.8)$ & $145(58.7)$ & $7(2.8)$ \\
\hline
\end{tabular}

was frequently cited for rectus muscle reapproximation and skin closure techniques. "Concerns for increasing future adhesions" was the top choice among those who close the parietal peritoneum, the only situation in which this response appears as a top three choice.

The most frequently chosen response about a specific surgical technique overall was not to perform single-layer hysterotomy closure among women planning future children, something that $73.3 \%$ reported they were less likely to perform (-Table 2); $70.4 \%$ of surgeons reported choosing their answer based on concern for future uterine rupture, with "Evidence-based" as the second most frequent response for this technique ( - Table 4). Suturing of the skin due to both better cosmetic result and patient preference were the next most frequently chosen reasons (159 responses; $64.4 \%$ each).

When asked to choose from a given list, the most important techniques to prevent adhesions, surgeons reported the following in order of frequency: parietal peritoneum closure (42.2\%), double-layer hysterotomy closure (29.6\%), reapproximation of the rectus muscles (19.9\%), and visceral peritoneum closure (13.4\%).

\section{Discussion}

Our study demonstrates a lack of consistency in the practices of rectus muscle reapproximation and parietal peritoneum closure at CD among survey respondents in the United States, with similar numbers of obstetricians practicing each technique (38\% reapproximate rectus muscles, $43 \%$ do not; $43 \%$ close the parietal peritoneum, $47 \%$ do not). In contrast, we found that double-layer hysterotomy closure and suturing of the skin are commonly practiced, and closure of the visceral peritoneum is uncommon. Significant differences were seen in practice styles based on age and years in practice. Older doctors with more years in practice were more likely to close the parietal peritoneum and the visceral peritoneum and to reapproximate the rectus muscles; doctors with fewer years in practice were more likely to close the hysterotomy in two layers and to suture the skin. Most surgeons report that their practice style reflects primarily how they were trained.

Evidence-based guidelines exist for some, but not all, of the surgical closure techniques we queried. Dahlke et al updated a systematic review from Berghella et al, ${ }^{9,11}$ including randomized 
Table 3 Surgical techniques by average respondent age and years in practice ${ }^{a}$

\begin{tabular}{|l|l|l|l|l|}
\hline & "Always" or “Usually" & "Sometimes" & "Rarely" or "Never" & $p$ Value \\
\hline Perform a single-layer hysterotomy & & & & \\
\hline Age & $49.7 \pm 1.7(38)$ & $50.6 \pm 3.1(22)$ & $47.7 \pm 0.7(181)$ & 0.328 \\
\hline Years in practice & $18.5 \pm 1.7(39)$ & $19.0 \pm 3.1(22)$ & $15.0 \pm 0.8(180)$ & 0.069 \\
\hline Close the parietal peritoneum & & & & \\
\hline Age & $50.3 \pm 1.0(105)$ & $48.7 \pm 2.0(23)$ & $46.5 \pm 1.0(115)$ & 0.028 \\
\hline Years in practice & $18.1 \pm 1.1(105)$ & $15.5 \pm 2.2(22)$ & $14.1 \pm 1.0(116)$ & 0.025 \\
\hline Close the visceral peritoneum & & & & \\
\hline Age & $55.7 \pm 1.4(30)$ & $55.0 \pm 2.9(14)$ & $46.4 \pm 0.7(193)$ & $<0.001$ \\
\hline Years in practice & $24.3 \pm 1.4(30)$ & $22.9 \pm 3.1(14)$ & $13.8 \pm 0.7(193)$ & $<0.001$ \\
\hline Reapproximate the rectus muscles & & & & \\
\hline Age & $50.7 \pm 1.0(95)$ & $46.6 \pm 1.5(41)$ & $46.8 \pm 1.1(106)$ & 0.016 \\
\hline Years in practice & $18.3 \pm 1.1(95)$ & $14.4 \pm 1.6(40)$ & $14.5 \pm 1.1(107)$ & 0.023 \\
\hline Suture the skin & & & & \\
\hline Age & $47.7 \pm 0.8(167)$ & $47.7 \pm 2.3(31)$ & $51.0 \pm 1.4(42)$ & 0.174 \\
\hline Years in practice & $15.1 \pm 0.8(166)$ & $15.3 \pm 2.4(31)$ & $19.3 \pm 1.5(43)$ & 0.072 \\
\hline Staple the skin & & & & \\
\hline Age & $50.9 \pm 1.4(55)$ & $47.3 \pm 1.9(39)$ & $47.7 \pm 0.8(145)$ & 0.121 \\
\hline Years in practice & $18.8 \pm 1.5(56)$ & $14.4 \pm 1.8(39)$ & $15.2 \pm 0.9(144)$ & 0.066 \\
\hline
\end{tabular}

${ }^{a}$ Years, mean \pm standard error of the mean, and $(n)$ where indicated.

${ }^{\mathrm{b}} p$ Values were calculated using analysis of variance.

controlled trials, meta-analyses or systematic reviews, and Cochrane reviews, most of which focus on short-term outcomes, and made evidence-based recommendations using the U.S. Preventative Services Task Force definitions. No recommendation could be made regarding rectus muscle reapproximation due to insufficient evidence. Indeed, no randomized controlled trials of rectus reapproximation have been published. When the search terms "rectus," "muscle," and "cesarean" or "rectus," "cesarean," and "closure" were entered into PubMed, utilizing the English language literature from 1950 to the present, only one result about rectus muscle reapproximation was returned, ${ }^{6}$ a prospective cohort study that suggested a decrease in adhesions with reapproximation. Given the limited data about rectus muscle reapproximation, the lack of consistency in practice styles is likely to continue.

Data are limited regarding optimal surgical techniques at $\mathrm{CD}$ to reduce postsurgical adhesions, ${ }^{6}$ given the difficulty of conducting a long-term $\mathrm{CD}$ follow-up study. With regard to peritoneal closure, several studies compared a combined closure of the parietal and visceral peritoneum to nonclosure, ${ }^{14-17}$ so may not answer the question of peritoneum closure and adhesions, as some studies suggest that closure of the parietal peritoneum may decrease adhesions while closure of the visceral peritoneum may increase them. ${ }^{4,6,18}$ Dahlke et al concluded that based on limited data, parietal peritoneal closure may decrease the risk of future adhesions. ${ }^{9}$

Few (16\%) of respondents reported routinely closing the hysterotomy in one-layer among women planning future children, and $73 \%$ reported rarely taking this approach. In their systematic review, Dahlke et al acknowledge that data on uterine rupture are derived from cohort and case control studies, not randomized controlled trials. ${ }^{9}$ Two randomized controlled trials examining this question, the CORONIS and CAESAR collaboratives, are currently ongoing. ${ }^{14,15}$ Without a randomized controlled trial, the majority of U.S. obstetricians have chosen a more conservative approach and practice double-layer hysterotomy closure. Of note, we did not query respondents about their hysterotomy closure technique for women not planning future children as the question of uterine rupture was the factor underlying our question.

The majority of respondents (68\%) typically suture the skin, style unspecified, compared with $23 \%$ who typically use staples. According to the systematic review by Dahlke et al, ${ }^{9}$ staple closure was associated with a twofold greater risk of wound infection or separation compared with subcuticular closure, though a Cochrane review concluded that wound complications were similar with each technique. ${ }^{19}$ Based on this difference, Dahlke et al concluded that a definitive recommendation is difficult due to uncertainty. ${ }^{9}$

Two similar surveys of surgical techniques at CD conducted by Demers et al and Tully et al also demonstrated significant variation in closure techniques. $^{12,13}$ In a survey of 176 obstetrician-gynecologists conducted in Quebec, Canada, respondents similarly reported a lack of consistency in the practice closure of the parietal peritoneum (49\% close, 50\% do not close), reported that most do not close the visceral peritoneum (17\% close, $81 \%$ do not close) and that most close the hysterotomy in two layers ( $89 \%$ versus $10 \%$ single layer). ${ }^{13}$ In 


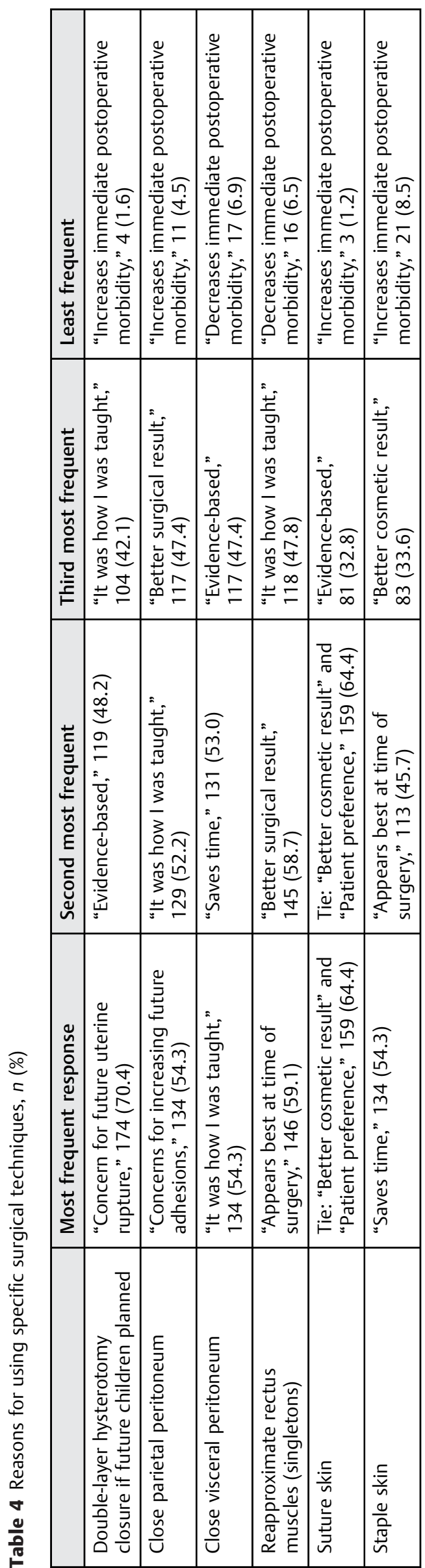

contrast to our results, fewer respondents reapproximate the rectus muscles ( $27 \%$ close, $72 \%$ do not close), and more close the skin with staples ( $86 \%$ versus $14 \%$ use suture). In a 1999 survey of 2,374 members of the Royal College of Obstetricians and Gynaecologists, Tully et al found that, compared with our results, fewer surgeons closed the parietal peritoneum (34\% versus $66 \%$ did not close), more closed the visceral peritoneum ( $28 \%$ versus $72 \%$ did not close), and nearly all closed the hysterotomy in two layers (94\% versus 3\% single layer); rectus muscle reapproximation was not mentioned. ${ }^{13}$

Our study has several strengths. Our survey was sent to a large number of ACOG members, included practitioners from all practice environments, and should be reflective of contemporary practice. In contrast to published systematic reviews on evidence-based surgical techniques at $\mathrm{CD}$, our study addresses a unique question that is otherwise to date unaddressed in the United States: what surgical practices do obstetricians actually employ at CD? Our study is limited by potentially inherent and difficult to measure biases reflected in any survey, such as whether there may be practice differences among those who chose to respond, and differences among those who use e-mail, as those not using e-mail were not included. We sent the survey to many who no longer practice obstetrics, as there was no way to identify in those who do not practice.

$\mathrm{CD}$ is the most common surgical procedure performed in women in the United States, yet similar to surveys conducted in Canada and Britain, our survey demonstrated significant variation in surgical closure techniques. Age, years in practice, and how one was trained are some of the reasons for such variation. The lack of a strong evidence base for techniques to minimize long-term, and in some cases short-term, morbidity likely also plays a role in the observed practice variation. The prospective randomized trials currently being conducted through the CORONIS and CAESAR collaboratives may answer some questions about optimal surgical techniques, particularly with regard to hysterotomy closure. ${ }^{14,15}$

Given the limited level 1evidence for many closure techniques at $\mathrm{CD}$, it is likely current practices will remain unchanged until a stronger evidence base exists.

\section{Acknowledgments}

This research is supported by research grant HRSA UA6MC19010.

\section{References}

1 Martin JA, Hamilton BE, Osterman MJK, et al. Births: final data for 2012. National Vital Statistics Reports. Vol. 62. No. 9. Hyattsville, MD: National Center for Health Statistics; 2013

2 Irion O, Luzuy F, Béguín F. Nonclosure of the visceral and parietal peritoneum at caesarean section: a randomised controlled trial. $\mathrm{Br}$ J Obstet Gynaecol 1996;103(7):690-694

3 Nagele F, Karas H, Spitzer D, et al. Closure or nonclosure of the visceral peritoneum at cesarean delivery. Am J Obstet Gynecol 1996;174(4):1366-1370

4 Lyell DJ, Caughey AB, Hu E, Daniels K. Peritoneal closure at primary cesarean delivery and adhesions. Obstet Gynecol 2005;106(2): 275-280 
5 Lyell DJ. Adhesions and perioperative complications of repeat cesarean delivery. Am J Obstet Gynecol 2011;205(6, Suppl):S11-S18

6 Lyell DJ, Caughey AB, Hu E, Blumenfeld Y, El-Sayed YY, Daniels K. Rectus muscle and visceral peritoneum closure at cesarean delivery and intraabdominal adhesions. Am J Obstet Gynecol 2012; 206(6):515.e1-515.e5

7 Blumenfeld YJ, Caughey AB, El-Sayed YY, Daniels K, Lyell DJ. Singleversus double-layer hysterotomy closure at primary caesarean delivery and bladder adhesions. BJOG 2010;117(6):690-694

8 Greenberg MB, Daniels K, Blumenfeld YJ, Caughey AB, Lyell DJ. Do adhesions at repeat cesarean delay delivery of the newborn? Am J Obstet Gynecol 2011;205(4):380.e1-380.e5

9 Dahlke JD, Mendez-Figueroa H, Rouse DJ, Berghella V, Baxter JK, Chauhan SP. Evidence-based surgery for cesarean delivery: an updated systematic review. Am J Obstet Gynecol 2013;209(4):294-306

10 Hofmeyr JG, Novikova N, Mathai M, Shah A. Techniques for cesarean section. Am J Obstet Gynecol 2009;201(5):431-444

11 Berghella V, Baxter JK, Chauhan SP. Evidence-based surgery for cesarean delivery. Am J Obstet Gynecol 2005;193(5):1607-1617

12 Tully L, Gates S, Brocklehurst P, McKenzie-McHarg K, Ayers S. Surgical techniques used during caesarean section operations: results of a national survey of practice in the UK. Eur J Obstet Gynecol Reprod Biol 2002;102(2):120-126
13 Demers S, Roberge S, Afiuni YA, Chaillet N, Girard I, Bujold E. Survey on uterine closure and other techniques for Caesarean section among Quebec's obstetrician-gynaecologists. J Obstet Gynaecol Can 2013;35(4):329-333

14 The CORONIS Collaborative Group. CORONIS-International study of caesarean section surgical techniques: the follow-up study. BMC Pregnancy and Childbirth 2013;13:215

15 CAESAR study collaborative group. Caesarean section surgical techniques: a randomised factorial trial (CAESAR). BJOG 2010; 117(11):1366-1376

16 Roset E, Boulvain M, Irion O. Nonclosure of the peritoneum during caesarean section: long-term follow-up of a randomised controlled trial. Eur J Obstet Gynecol Reprod Biol 2003;108(1):40-44

17 Ghahiry A, Rezaei F, Karimi Khouzani R, Ashrafinia M. Comparative analysis of long-term outcomes of Misgav Ladach technique cesarean section and traditional cesarean section. J Obstet Gynaecol Res 2012;38(10):1235-1239

18 Woytoń J, Florjański J, Zimmer M. [Nonclosure of the visceral peritoneum during Cesarean sections]. Ginekol Pol 2000;71(10): 1250-1254 Polish.

19 Mackeen AD, Berghella V, Larsen ML. Techniques and materials for skin closure in caesarean section. Cochrane Database Syst Rev 2012;11:CD003577 